\title{
D-Ribose Induces Podocyte NLRP3 Inflammasome Activation and Glomerular Injury via AGEs/RAGE Pathway
}

\author{
Jinni Hong ${ }^{1,2,3}$, Guangbi Li ${ }^{3}$, Qinghua Zhang ${ }^{3}$, Joseph Ritter ${ }^{3}$, Weiwei Li' and Pin-Lan Li ${ }^{3 *}$ \\ ${ }^{1}$ Integrated Laboratory of Traditional Chinese Medicine and Western Medicine, Peking University First Hospital, Beijing, \\ China, ${ }^{2}$ Department of Gynecology, The Affiliated People's Hospital of Fujian University of Traditional Chinese Medicine, \\ Fuzhou, China, ${ }^{3}$ Department of Pharmacology and Toxicology, Medical College of Virginia, Virginia Commonwealth \\ University, Richmond, VA, United States
}

D-ribose levels are demonstrated to be increased in type II diabetes mellitus and increased blood D-ribose is involved in the development of diabetic complications such as diabetic encephalopathy and nephropathy. However, the mechanism mediating the pathogenic role of D-ribose in nephropathy remains poorly understood. Given

OPEN ACCESS

Edited by:

Zhengchao Wang,

Fujian Normal University, China

Reviewed by:

Xiaofeng Yang,

Temple University, United States

Mosharraf Sarker,

Teesside University, United Kingdom

Zhenghong Zhang,

Fujian Normal University, China

*Correspondence:

Pin-Lan Li

pin-lan.li@vcuhealth.org

Specialty section:

This article was submitted to

Molecular Medicine,

a section of the journal

Frontiers in Cell and Developmental

Biology

Received: 25 August 2019 Accepted: 17 October 2019 Published: 30 October 2019

Citation:

Hong J, Li G, Zhang Q, Ritter J,

Li W and Li P-L (2019) D-Ribose

Induces Podocyte NLRP3

Inflammasome Activation

and Glomerular Injury via AGEs/RAGE

Pathway. Front. Cell Dev. Biol. 7:259.

doi: 10.3389/fcell.2019.00259 that D-ribose was reported to induce advanced glycation end products (AGEs) formation, the present study tested whether D-ribose induces NLRP3 activation and associated glomerular injury via AGEs/receptor of AGEs (RAGE) signaling pathway. In vivo, C57BL/6J and $\mathrm{Asc}^{-/-}$mice were treated with D-ribose with or without AGEs inhibitor. Administration of D-ribose daily for 30 days was found to induce NLRP3 inflammasome formation in glomerular podocyte, as shown by increased co-localization of NLRP3 with apoptosis-associated speck-like protein containing a caspase recruitment domain (ASC) or caspase-1. This D-ribose-induced NLRP3 inflammasome formation was accompanied by its activation as evidenced by increased IL-1 $\beta$ production, a major product of NLRP3 inflammasome. Corresponding to NLRP3 inflammasome activation, D-ribose led to significant glomerular injury in mice. All these D-ribose-induced glomerular inflammasome and associated pathological changes were markedly attenuated by deletion of Asc gene. Furthermore, the accumulation of AGEs and RAGE was found increased in glomeruli of mice receiving D-ribose. In cell studies, we also confirmed that D-ribose induced NLRP3 inflammasome formation and activation in podocytes, which was significantly blocked by caspase-1 inhibitor, YVAD. Mechanically, AGEs formation inhibition and cleavage or silencing of RAGE gene were shown to suppress D-ribose-induced NLRP3 inflammasome formation and activation, as shown by significant reduction of NLRP3 inflammasome molecular aggregation, caspase- 1 activity and IL-1 $\beta$ production. These results strongly suggest that relatively long term administration of D-ribose induces NLRP3 inflammasome formation and activation in podocytes via AGEs/RAGE signaling pathway, which may be one of important triggering mechanisms leading to diabetic nephropathy.

Keywords: pentose monosaccharide, inflammatory machinery, AGEs-RAGE system, glomerular disease, endstage renal disease 


\section{INTRODUCTION}

D-ribose is a naturally occurring monosaccharide present in all living cells. It is mainly supplied in food and widely recommended as a supplement of metabolic therapy for chronic fatigue syndrome and coronary artery disease (Perlmutter et al., 1991; Teitelbaum et al., 2006). The beneficial effect of D-ribose is to lower malondialdehyde and glutathione levels during hypoxic stress (Gross et al., 1989; Seifert et al., 2009). However, a potential adverse effect of ribosylation was raised recently. The nonenzymatic glycation of proteins by reduction of D-ribose may lead to fructosamine formation (Day et al., 1979) and irreversible production of advanced glycation end products (AGEs), the toxic glycated proteins (Wei et al., 2009). Recently, D-ribose as a potential risk factor in the development of type II diabetes mellitus (T2DM) has been considered being overlooked ( $\mathrm{Su}$ and $\mathrm{He}, 2014$ ), given that D-ribose produces AGEs much more rapidly than D-glucose (Chen et al., 2009; Wei et al., 2009). Patients with diabetes mellitus (DM) suffering from metabolic derangements and organ complications may be associated not only with D-glucose but also with D-ribose (Babaei-Jadidi et al., 2003; Wei et al., 2009, 2012; Lopez-Clavijo et al., 2014; Tessier et al., 2016). It has been reported that in T2DM patients, blood and urine D-ribose levels were increased, which may trigger tissue inflammation, Tau protein hyper-phosphorylation, and amyloid $\beta$-like deposits in brain, resulting in cognitive impairment (Han et al., 2011, 2014; Wu et al., 2015). In a recent study, we also found that D-ribose induced glomerular inflammation and sclerosis with increased AGEs level in mouse kidney. However, it remains unknown how D-ribose induces glomerular inflammation and leads to glomerular injury.

Accumulating evidence shows that the formation and activation of Nod-like receptor protein 3 (NLRP3) inflammasome in podocytes may instigate glomerular inflammation and lead to ultimate glomerular sclerosis in response to pathological stimuli such as hyperhomocysteinemia (hHcy), obesity and DM (Yi et al., 2006; Abais et al., 2013, 2014a). The NLRP3 inflammasome serves as an intracellular inflammatory machinery, which can be activated by a wide range of danger signals and lead to various chronic degenerative diseases (Zhang et al., 2012; Yin et al., 2013; Bakker et al., 2014). This novel cytosolic multiprotein complex is composed of NLRP3, the adaptor molecule apoptosis-associated speck-like protein containing a CARD (caspase recruitment domain) (ASC), and the cysteine protease caspase-1. During formation and activation of the NLRP3 inflammasome, cleaved caspase-1 causes the maturation of pro-inflammatory cytokines, such as IL-1 $\beta$ and IL18. As a canonical mechanism, secretion of these inflammatory cytokines to extracellular space triggers tissue inflammatory response (Martinon and Tschopp, 2005). Furthermore, NLRP3 inflammasome activation may also induce cell metabolic disturbance, cell transformation, and tissue damage as its uncanonical effects, which may be another important triggering mechanism for organ diseases beyond inflammation (Yang et al., 2014). To our knowledge, so far there is no study that links the detrimental effects of D-ribose to NLRP3 inflammasome activation in glomerular injury or sclerosis.
The present study was designed to test the hypothesis that D-ribose induces NLRP3 inflammasome formation and activation in podocytes in an AGEs dependent manner, which will be an important triggering mechanism leading to podocyte injury and glomerular sclerosis. We first demonstrated that D-ribose induced podocyte injury and glomerular sclerosis in wide-type (WT) mice compared with Asc gene knockout mice. We also observed whether the pathological role of D-ribose to induce NLRP3 inflammasome activation is associated with increased production of AGEs. Then, we performed in vitro studies to test whether D-ribose-induced NLRP3 inflammasome activation is associated with AGEs-RAGE signaling pathway. These studies together confirmed the key role of NLRP3 inflammasome activation in D-ribose-induced podocyte injury and consequent glomerular sclerosis, which is mediated by AGEs-RAGE signaling pathway.

\section{MATERIALS AND METHODS}

\section{Animals}

Eight-week-old, male C57BL/6J (The Jackson Laboratory, Bar Harbor, ME, United States) were intraperitoneally (i.p.) injected vehicle or D-ribose (dissolved in $0.9 \%$ saline) at a dose of $2 \mathrm{~g} / \mathrm{kg} \mathrm{BW}$, once a day, for 30 days. In another series, male $\mathrm{ASC}^{-/-}$mice and their wild-type littermates at the same age were used for confirmation of NLRP3 inflammasome involvement in the action of D-ribose. All mice were randomly distributed to Vehl (Vehicle), D-R (D-ribose) and D-R + AG (D-ribose + aminoguanidine, AGEs formation inhibitor) groups, 8 mice in each group. Mice of $\mathrm{D}-\mathrm{R}+\mathrm{AG}$ group were additionally fed with $1 \mathrm{~g} / \mathrm{L}$ AG in water for 30 days (Yavuz et al., 2001). All mice were housed under identical conditions in a pathogen-free environment with a 12:12 h light/dark cycle and free access to laboratory chow and water. Mice were acclimatized to the housing environment for at least 1 week before the experiments. 3 days before the protocol was finished, mice were placed in metabolic cages to collect urine samples for analysis of urinary albumin and protein excretion. On the day protocol was completed, blood samples were taken for measurement of fasting blood glucose with OneTouch Ultra2 blood glucose meter (LifeScan Europe, Switzerland). Then, mice were sacrificed under mild ethyl ether anesthesia and their kidneys were harvested. All animal experimental protocols were approved by the Institutional Animal Care and Use Committee of the Virginia Commonwealth University.

\section{Cell Culture}

A conditionally immortalized mouse podocyte cell line (Graciously provided by Dr. P. E. Klotman, Division of Nephrology, Department of Medicine, Mount Sinai School of medicine, New York, NY, United States), were cultured and maintained as described before (Abais et al., 2013; Hong et al., 2019). For all experiments, culture medium was replaced with serum-free medium for $24 \mathrm{~h}$ prior to treatments. Podocytes were incubated with $25 \mathrm{mM}$ D-ribose (Sigma, United States), 
$25 \mathrm{mM}$ L-ribose (AK scientific, United States) as negative control (AK scientific, United States) and $25 \mathrm{mM}$ D-glucose (Sigma, United States) as positive control for $24 \mathrm{~h}$. To inhibit caspase-1 activity in podocytes, its selective inhibitor, AcYVAD-CMK (YvAD, $10 \mu \mathrm{g} / \mathrm{ml}$, Cayman Chemical) was used $30 \mathrm{~min}$ prior to treatments. To inhibit the role of AGEs, AGEs formation inhibitor aminoguanidine (AG, $50 \mu \mathrm{M}$, Sigma Aldrich) and a breaker of AGEs-based crosslinks, alagebrium chloride (ALT, $100 \mu \mathrm{M}$, TCI AMERICA) were used $30 \mathrm{~min}$ prior to treatments (Dhar et al., 2016; Chowdhury, 2017).

\section{Glomerular Morphological Examinations}

Kidneys were fixed with $4 \%(\mathrm{v} / \mathrm{v})$ paraformaldehyde (PFA) in PBS, embedded with paraffin, sliced into $4 \mu \mathrm{m}$ sections and stained with Periodic Acid-Schiff. Glomerular morphology was observed and assessed semi-quantitatively as described previously (Raij et al., 1984; Abais et al., 2014b).

\section{Urinary Protein and Albumin Measurements}

Total urinary protein concentrations were determined spectrophotometrically using Bradford assay (Sigma, United States). Urinary albumin concentration was measured with mouse albumin ELISA kit (Bethyl Laboratories, Montgomery, TX, United States) according to manufacturer's instructions.

\section{Immunohistochemistry}

After embedded and sectioned, slides were incubated with primary antibody against IL-1 $\beta$ (1:200, R\&D Systems, United States), RAGE (1:200, Sigma, United States) and AGEs (1:200, Abcam, Cambridge, MA, United States) at $4^{\circ} \mathrm{C}$ overnight. Then slides were incubated with biotinylated secondary antibodies and a streptavidin peroxidase complex (PK-7800, Vector Laboratories, Burlingame, CA, United States). Finally, samples observed with microscopy as described previously (Raij et al., 1984; Hong et al., 2019). The area percentage of the positive staining was calculated with Image Pro Plus 6.0 software (Raij et al., 1984).

\section{Immunofluorescence Microscopy}

After treatments, kidney slides and podocyte culture coverslips were fixed, blocked and incubated with primary antibodies against NLRP3 (1:100, Abcam, Cambridge, MA, United States), ASC (1:200, Santa Cruz Biotechnology, Dallas, TX, United States), cleaved-caspase-1 (1:200, Santa Cruz Biotechnology, Dallas, TX, United States), podocin (1:400, Sigma), or desmin (1:400, Thermo Fisher Scientific) at $4^{\circ} \mathrm{C}$ overnight. Then slides were incubated with corresponding second antibodies with either Alexa- 488- or Alexa-555-labeled (Invitrogen, Carlsbad, CA, United States). For example, slides incubated with NLRP3 were then incubated with donkey anti goat secondary antibody, Alexa fluor plus 488, slides incubated with ASC, cleaved-caspase-1 or Podocin were then incubated with donkey anti mouse secondary antibody,
Alexa fluor plus 555, slides incubated with desmin were then incubated with donkey anti rabbit secondary antibody, Alexa fluor plus 488. After that, slides were observed with a laser scanning confocal microscope (Fluoview FV1000, Olympus, Japan). Co-localization coefficient was analyzed with Image Pro Plus 6.0 software and presented by Pearson's correlation coefficient (PCC).

\section{Western Blot Analysis}

Equivalent amount of proteins (20-30 $\mu \mathrm{g}$ ) was resolved on SDS-PAGE gels and transferred to PVDF membrane. After blocking, membranes were probed with primary antibodies rabbit anti-Cle-Caspase-1 (1:1000, cell signaling technology), rabbit anti-pro-Caspase-1 (1:1000, Abcam, Cambridge, MA) and rabbit anti- $\beta$-actin (1:10000, Santa Cruz Biotechnology, Dallas, TX, United States) at $4^{\circ} \mathrm{C}$ overnight. After incubated with donkey anti-rabbit-HRP IgG (1:5000, Santa Cruz Biotechnology, Dallas, TX, United States), immunoreactive bands were detected by chemiluminescence techniques with LI-COR Odyssey Fc. The intensity of the specific bands was calculated with ImageJ software (NIH, Bethesda, MD, United States).

\section{Assays of Caspase-1 Activity and IL-1 $\beta$ Production}

Caspase-1 activity was measured with a commercial colorimetric assay kit (Biovision, Mountain View, CA, United States), with OD $=405 \mathrm{~nm}$, and IL-1 $\beta$ production in cell supernatant was detected with mouse IL- $1 \beta$ ELISA kit ( $R \& D$ systems, United States), with $\mathrm{OD}=450 \mathrm{~nm}$, according to manufacturer's instructions.

\section{RNA Interference of RAGE}

RAGE small interference RNAs (siRNAs) was purchased from Santa Cruz Biotechnology, Dallas, TX, United States. SiRNAs transfection was performed with the silent Lipid Reagent (BioRad, United States) according to manufacturer's instructions.

\section{Statistical Analysis}

Data are presented as means \pm SE. The significant differences between and within multiple groups were examined using one way or two-way ANOVA, followed by Duncan's multiple-range test. $P<0.05$ was considered statistically significant.

\section{RESULTS}

\section{D-Ribose Induced Glomerular Dysfunction and Injury in an AGEs-Dependent Manner}

We first tested whether D-ribose treatment induced podocyte injury and glomerular damage in WT and Asc gene knockout mice. As shown in Figures 1A,B, i.p. injection of D-ribose for 30 days induced proteinuria and albuminuria in WT mice in comparison with mice treated with vehicle, which were markedly blocked by simultaneous administration of AG, 

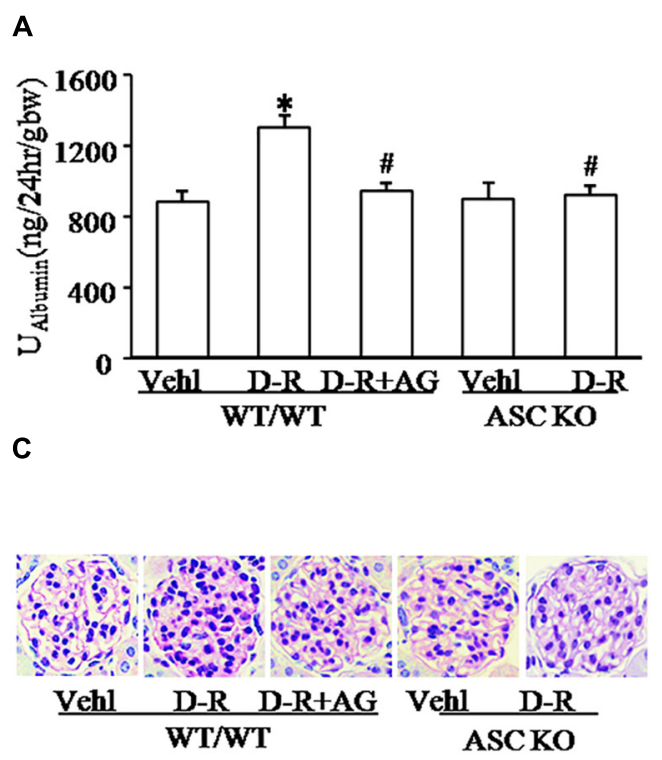

E

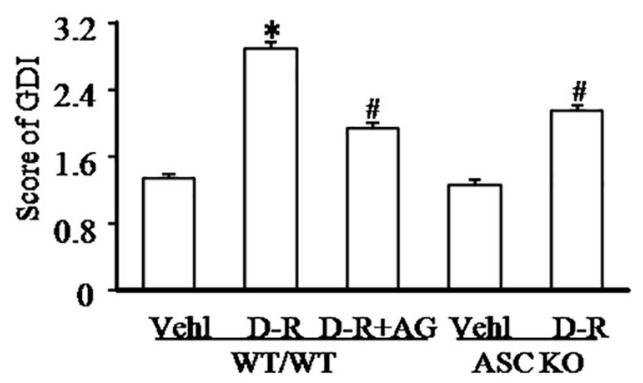

B

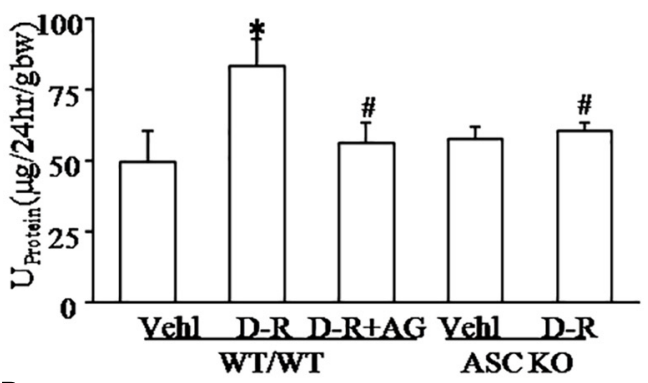

D
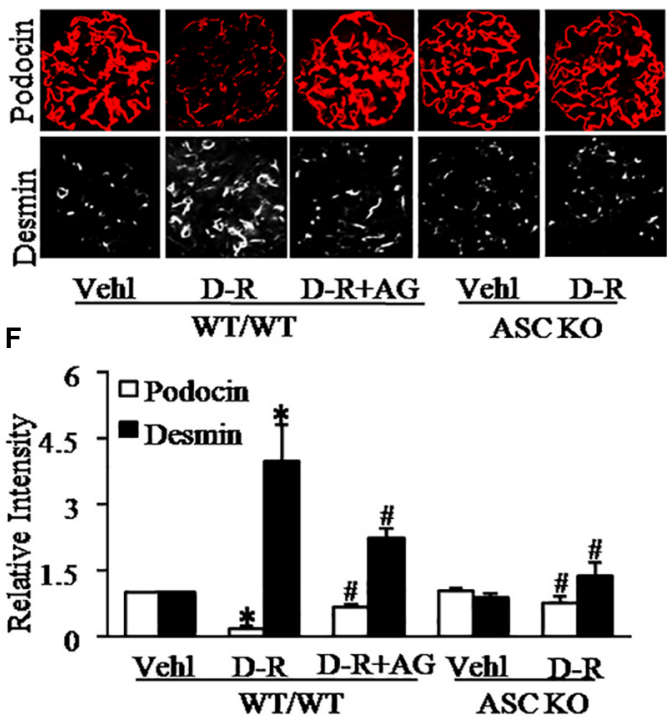

FIGURE 1 | D-ribose induced glomerular dysfunction and injury in an AGEs-dependent manner. (A) Urine albumin excretion of mice with different genotypes and treatment $(n=6)$. (B) Urine protein excretion of mice with different genotypes and treatment $(n=6)$. (C) Representative images of glomerular morphological changes (Periodic Acid-Schiff staining) from mice with different genotypes and treatments $(n=5)$. (D) Representative images of immunostained glomeruli for podocin and desmin from mice with different genotypes and treatments $(n=5)$. (E) Summarized data showing glomerular damage index (GDI) (Periodic Acid-Schiff staining) from mice with different genotypes and treatments $(n=5)$. (F) Summarized data of immunostained glomeruli for podocin and desmin from mice with different genotypes and treatments $(n=5)$. ${ }^{*} P<0.05$ versus WTMT-Vehl group, ${ }^{\#} P<0.05$ versus WTMT-D-R group. Vehl, Vehicle; D-R, D-ribose; AG, Aminoguanidine.

an AGEs formation inhibitor. In Asc gene knockout mice, D-ribose-induced proteinuria and albuminuria were much less severe. Consistently, WT mice receiving D-ribose injection had remarkable extracellular matrix and collagen deposition, mesangial cell expansion and capillary collapse, indicating a typical glomerular sclerotic pathology (Figures 1C,E), which were abolished by AG co-treatment. Using confocal microscopy, D-ribose was found to markedly alleviate podocin staining, but increase the fluorescence intensity of desmin staining (Figures 1D,F) in glomeruli of WT mice. After injection of D-ribose for 30 days, fasting-blood glucose in D-ribose-treated mice was significantly decreased compared to Vehl group (128.33 $\mathrm{mg} / \mathrm{dL}$ vs. $161.83 \mathrm{mg} / \mathrm{mL})$. All these glomerular and podocyte injuries induced by D-ribose were substantially blocked by administration of AG. Additionally, in Asc gene knockout mice, D-ribose treatment failed to induce podocyte injury and glomerular damage, as shown by no differences in all measured glomerular injurious parameters between mice receiving Vehl and D-ribose treatments.

\section{Attenuation of D-Ribose-Induced NLRP3 Inflammasome Activation by AG in Glomeruli}

As depicted in Figure 2A, the confocal microscopic analysis demonstrated that D-ribose remarkably increased co-localization of NLRP3 with ASC or caspase-1 (increased yellow staining) in glomeruli compared with Vehl group, which was blocked by AG treatment. Quantitation of the NLRP3 co-localization shows that NLRP3 inflammasome formation only occurred in mice receiving D-ribose, but not in mice of Vehl group (Figure 2B). Based on our previous studies showing that NLRP3 molecules are most enriched in podocytes, these results indicate the formation of NLRP3 inflammasome in glomerular podocytes of mice receiving D-ribose. Consistently, IL-1 $\beta$ as 

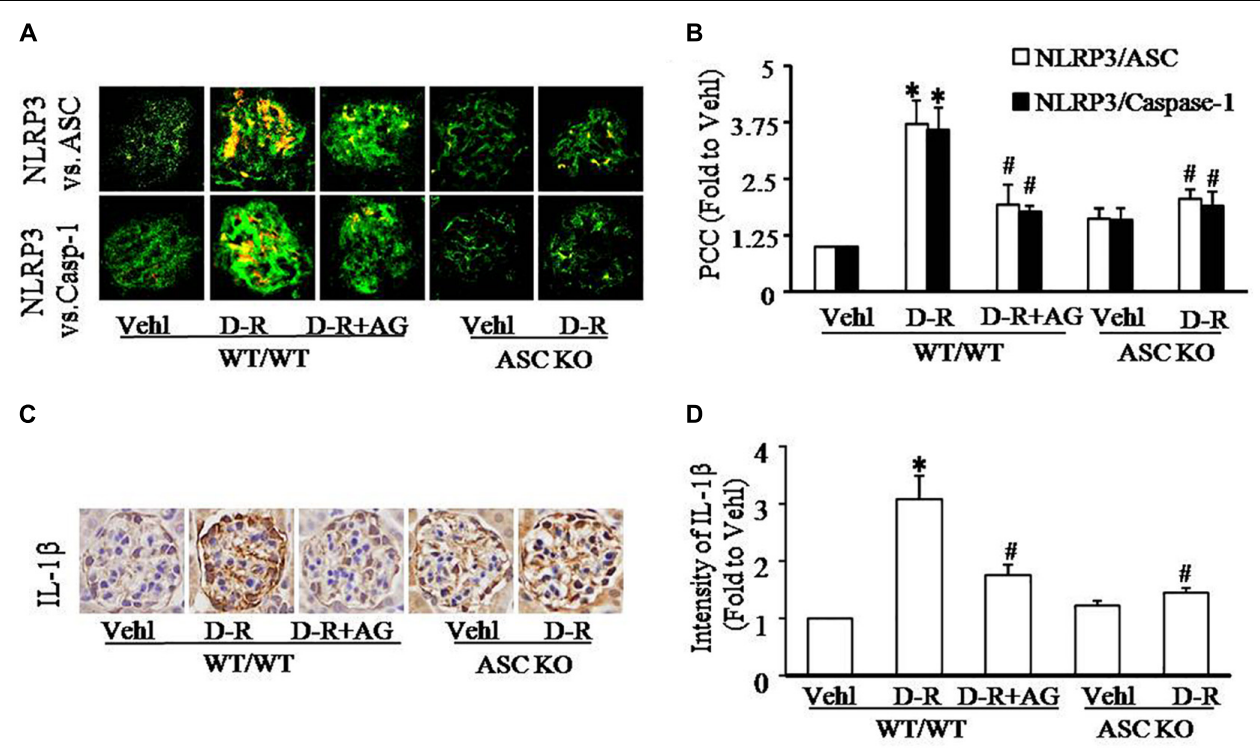

FIGURE 2 | D-ribose-induced NLRP3 inflammasome formation and activation in glomeruli. (A) Representative confocal microscopic images showing co-localization of NLRP3 (green) with ASC (red) or with caspase-1 (red) from mice with different genotypes and treatments $(n=5)$. (B) Summarized data showing co-localization of NLRP3 (green) with ASC (red) or with caspase-1(red) in glomeruli from mice with different genotypes and treatment $(n=5)$. (C) Representative photomicrographs depicting immunohistochemical staining of glomeruli with anti-IL-1 $\beta$ antibody from mice with different genotypes and treatments $(n=5)$. (D) Summarized data of immunohistochemical staining of glomeruli for IL-1 $\beta$ level from mice with different genotypes and treatments $(n=5)$. ${ }^{*} P<0.05$ versus WT/WT-Vehl group, ${ }^{\#} P<0.05$ versus WTMT-D-R group. Vehl, Vehicle; D-R, D-ribose; AG, Aminoguanidine.
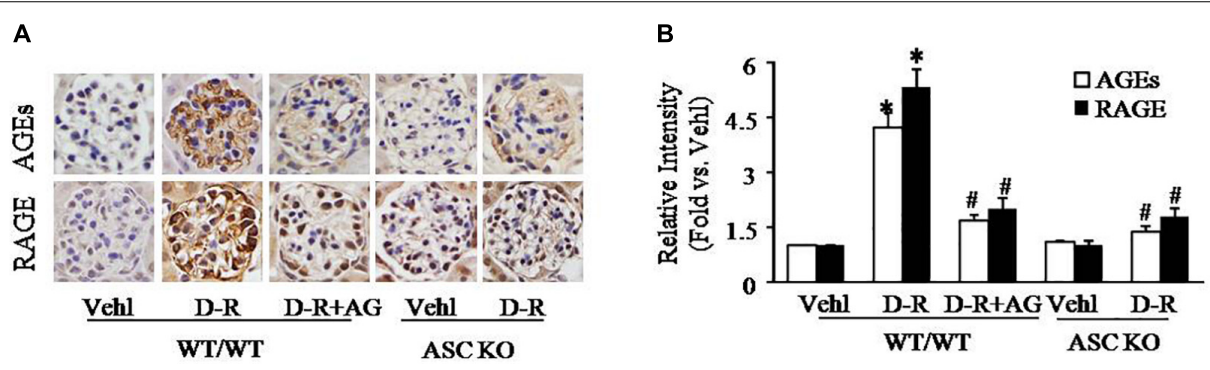

FIGURE 3 | Enhancement of AGEs production and RAGE expression in glomeruli of D-ribose-treated mice. (A) Representative photomicrograph showing immunohistochemical staining of glomeruli with anti-AGEs and RAGE antibodies in mice with different genotypes and treatments $(n=5)$. (B) Summarized data of immunohistochemical staining of glomeruli for AGEs and RAGE level from mice with different genotypes and treatments $(n=5)$. ${ }^{*}<0.05$ versus WT/WT-Vehl group, ${ }^{\#} P<0.05$ versus WTMT-D-R group. Vehl, Vehicle; D-R, D-ribose; AG, Aminoguanidine.

a prototype inflammatory cytokine produced by the NLRP3 inflammasome also significantly increased in glomeruli of mice treated with D-ribose compared to mice in Vehl group, which was markedly attenuated by AG (Figures 2C,D). In Asc gene knockout mice, however, D-ribose failed to induce NLRP3 inflammasome formation and activation in glomeruli. These findings demonstrate that NLRP3 inflammasome activation and consequent IL- $1 \beta$ production may contribute to D-riboseinduced podocyte injury and glomerular sclerosis.

\section{Enhancement of AGEs Production and RAGE Expression in Glomeruli of D-Ribose-Treated Mice}

To explore the mechanisms by which D-ribose induces NLRP3 inflammasome activation and podocyte injury, we observed changes in AGEs formation and RAGE expression in mouse glomeruli. By immunohistochemistry, AGEs and RAGE were remarkably elevated in glomeruli of mice receiving $\mathrm{D}$-ribose compared to Vehl group, and it could be totally blocked by AG, the AGEs formation inhibitor (Figures 3A,B).

\section{Effects of D-Ribose on NLRP3 Inflammasome Formation and Activation in Podocytes}

To further explore the mechanisms by which D-ribose activates NLRP3 inflammasome, we performed in vitro experiments in cultured podocytes. Caspase-1 inhibitor, YvAD, was used to pre-treat podocytes to test whether D-ribose indeed activates the NLRP3 inflammasome in podocytes in comparison with its isoform, L-ribose and positive control, D-glucose. By using 
A

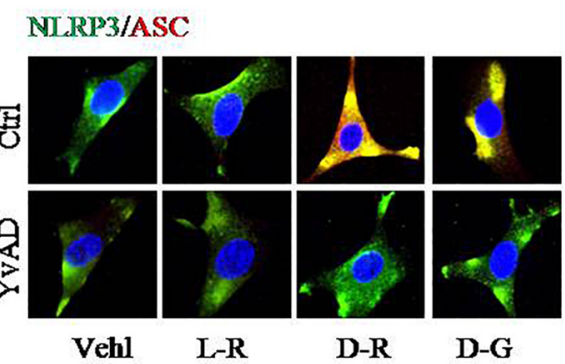

C

NILIRP3/ASC

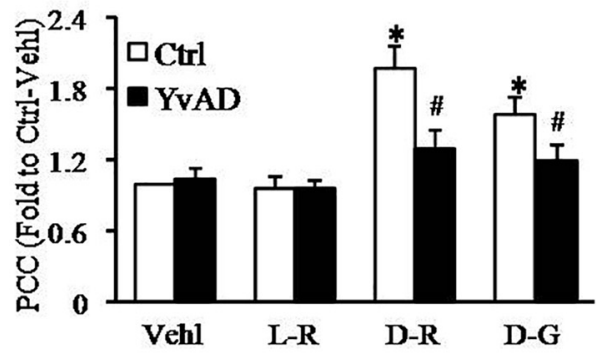

E

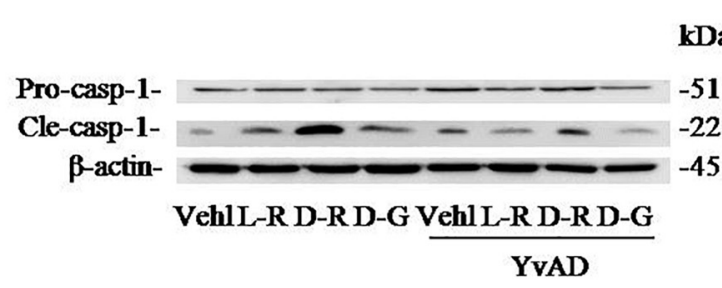

G

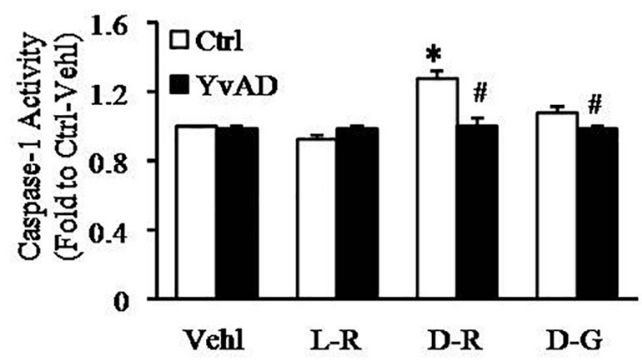

B

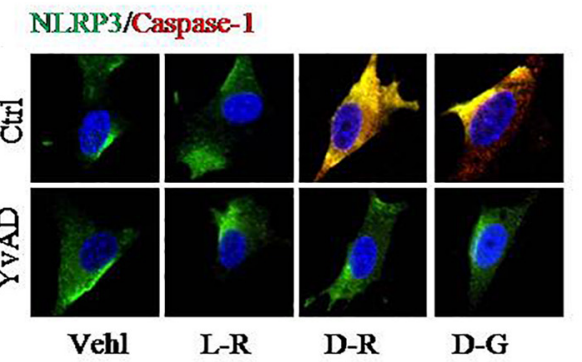

D

NLIRP3/Caspase-1

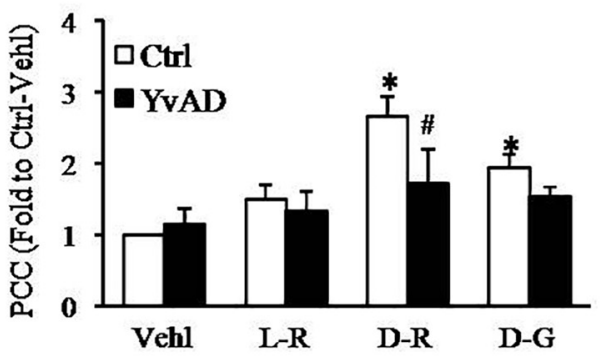

$\mathbf{F}$

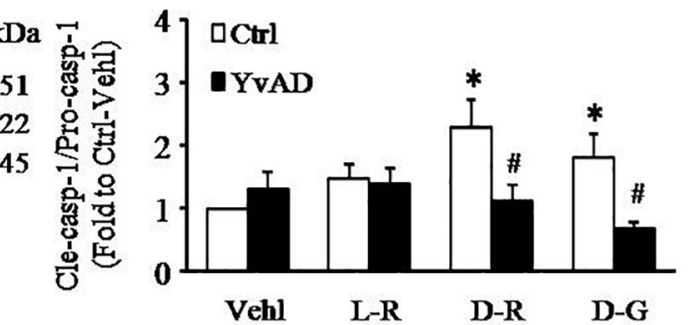

H

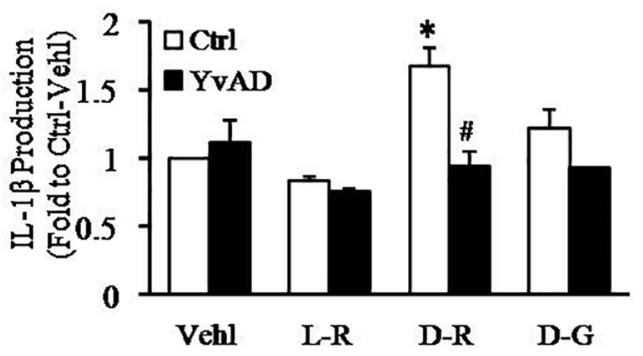

FIGURE 4 | Effects of D-ribose on NLRP3 inflammasome formation and activation in podocytes. (A) Representative confocal microscopic images showing co-localization of NLRP3 (green) with ASC (red) $(n=6)$. (B) Representative confocal microscopic images showing co-localization of NLRP3 (green) with caspase-1 (red) $(n=6)$. (C) Summarized data depicting co-localization of NLRP3 (green) with ASC (red) during different treatments of podocytes $(n=6)$. (D) Summarized data depicting co-localization of NLRP3 (green) with caspase-1 (red) during different treatments of podocytes $(n=6)$. (E) Representative Western blot gel document of pro-Caspase-1 and Cle-Caspase-1 in podocytes with different treatments $(n=5)$. (F) Summarized data of pro-Caspase-1 and Cle-Caspase- 1 in podocytes with different treatments $(n=5)$. (G) Caspase-1 activity in podocytes with different treatments $(n=6)$. (H) IL-1 $\beta$ levels in the supernatant of podocyte cultures with different treatments $(n=5) .{ }^{*} P<0.05$ versus Ctrl-Vehl group, ${ }^{\#} P<0.05$ versus Ctrl group. Ctrl, control; Vehl, Vehicle; L-R, L-ribose; D-R, D-ribose; D-G, D-glucose.

confocal microscopy, we demonstrated that co-localization of NLRP3 (green) with ASC (red) or caspase-1 (red), an indicative of NLRP3 inflammasome formation, was much higher in podocytes treated with D-ribose than Vehl and L-ribose, but similar to podocytes treated with D-glucose. However, prior treatment of podocytes with YvAD almost completely abolished the enhanced co-localization of NLRP3 with ASC or caspase-1 induced by D-ribose or D-glucose (Figures 4A,B). The colocalization coefficient was summarized and is shown below the representative confocal microscopic images (Figures 4C,D).

Based on previous studies, increased cleavage of caspase- 1 and enhanced caspase- 1 activity and consequent IL- $1 \beta$ production reflect NLRP3 inflammasome activation. The present study found that D-ribose treatment remarkably increased the level 
A

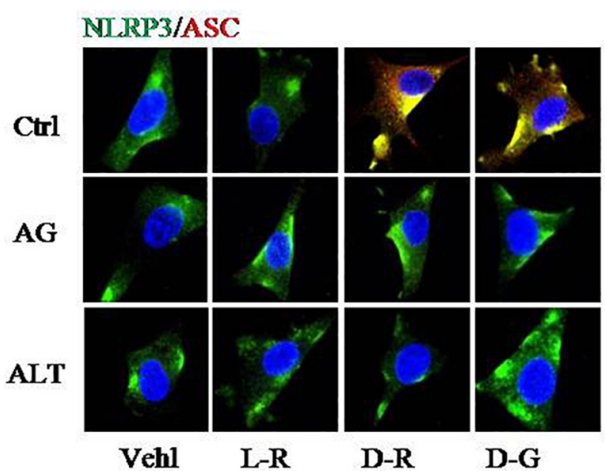

C

NITRP3/ASC

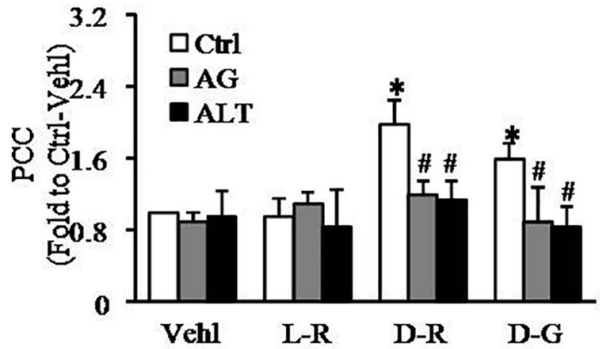

E

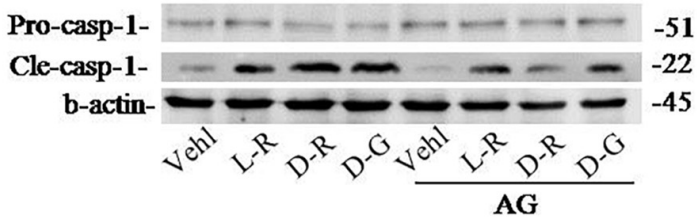

Pro-casp-1-

Cle-casp-1-

b-actin-

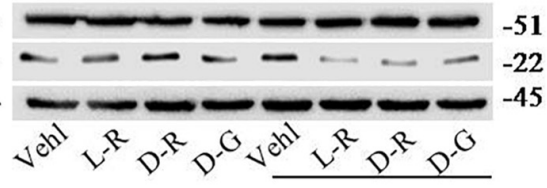

G

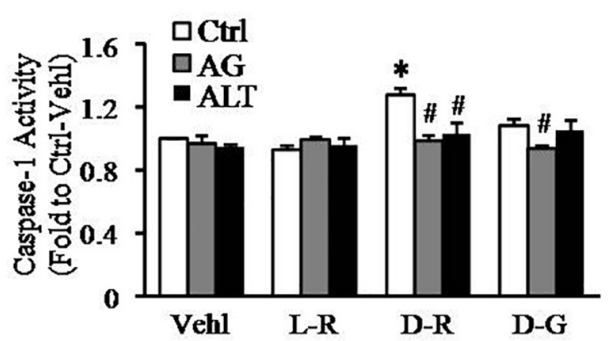

B NLIRP3/Caspase-1

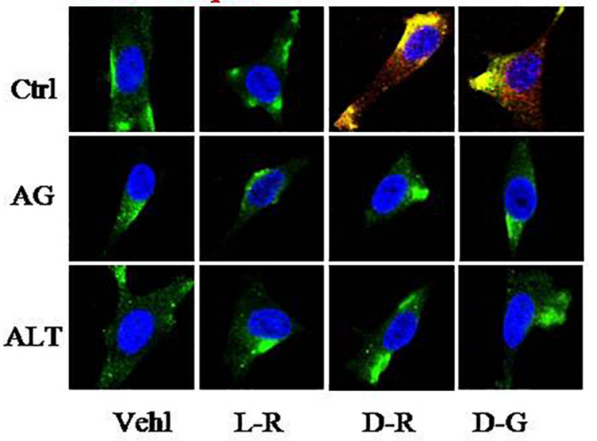

D NIRP3/Caspase-1

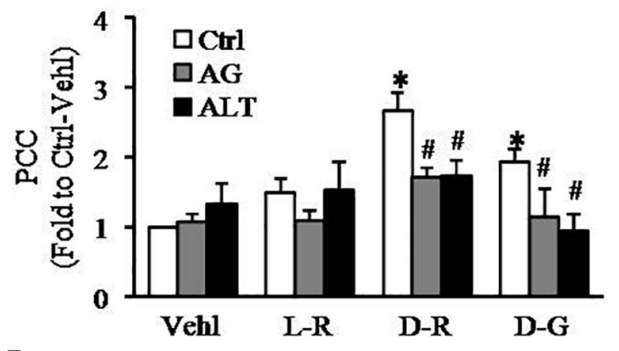

$\mathbf{F}$
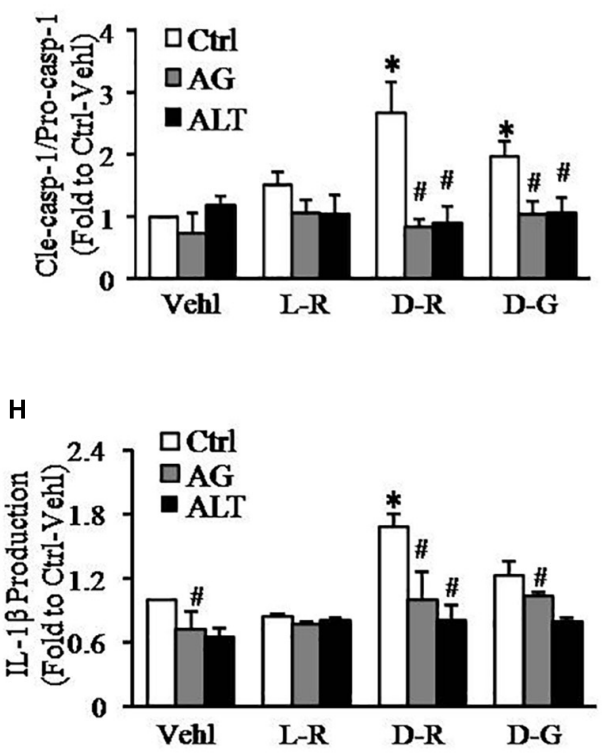

FIGURE 5 | Effects of AGEs formation inhibition and their crosslink breakers on D-ribose-induced NLRP3 inflammasome formation and activation in podocytes. (A) Representative confocal microscopic images showing co-localization of NLRP3 (green) with ASC (red) $(n=6)$. (B) Representative confocal microscopic images showing co-localization of NLRP3 (green) with caspase-1 (red) $(n=6)$. (C) Summarized data depicting co-localization of NLRP3 (green) with ASC (red) during different treatments of podocytes $(n=6)$. (D) Summarized data depicting co-localization of NLRP3 (green) with caspase-1 (red) during different treatments of podocytes $(n=6)$. (E) Representative Western blot gel document of pro- Caspase-1 and Cle-Caspase-1 in podocytes with different treatments $(n=5-6)$. (F) Summarized data of pro-Caspase-1 and Cle-Caspase-1 in podocytes with different treatments $(n=5-6)$. (G) Caspase-1 activity in podocytes with different treatments $(n=6)$. $(\mathbf{H}) \mathrm{IL}-1 \beta$ levels in the supernatant of podocyte cultures with different treatments $(n=5) .{ }^{*} P<0.05$ versus Ctrl-Vehl group, ${ }^{\#} P<0.05$ versus Ctrl group. Ctrl, control; Vehl, Vehicle; L-R, L-ribose; D-R, D-ribose; D-G, D-glucose. AG, aminoguanidine. ALT, alagebrium chloride.

of cleaved caspase-1 (22 kDa), but not procaspase-1(51 kDa) (Figures 4E,F), caspase-1 activity (Figure 4G) and IL-1 $\beta$ production (Figure $\mathbf{4 H}$ ), and prior treatment with YvAD almost completely attenuated these changes. Additionally, we demonstrated that D-glucose had D-ribose-like effect on NLRP3 inflammasome activation in podocytes. 
A

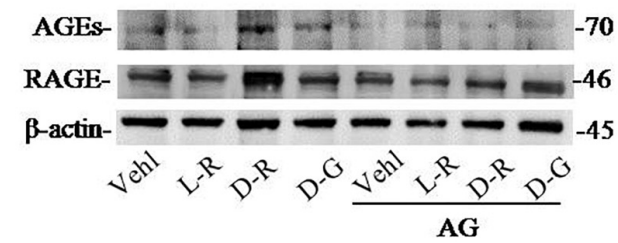

C

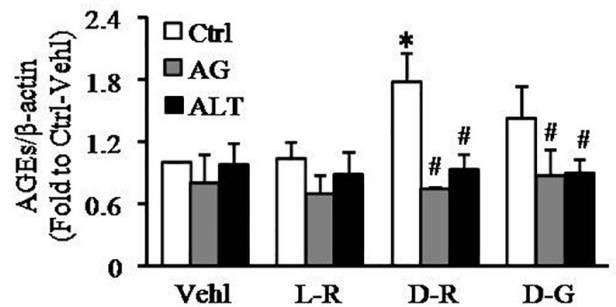

B

kDa

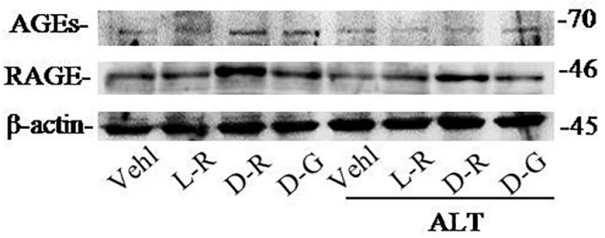

D

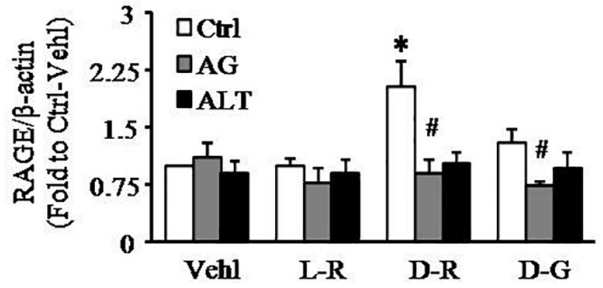

FIGURE 6 | AG and ALT blocked D-ribose-induced AGEs production and RAGE overexpression in podocytes. (A) Representative western blot gel document of AGEs and RAGE in podocytes with different treatments $(n=6)$. (B) Representative western blot gel document of AGEs and RAGE in podocytes with different treatments $(n=6)$. (C) Summarized data of AGEs in podocytes with different treatments $(n=6)$. (D) Summarized data of RAGE in podocytes with different treatments $(n=6) .{ }^{*} P<0.05$ versus Ctrl-Vehl group, \#P<0.05 versus Ctrl group. Ctrl, control; Vehl, Vehicle; L-R, L-ribose; D-R, D-ribose; D-G, D-glucose. AG, aminoguanidine. ALT, alagebrium chloride.

\section{Role of AGEs in D-Ribose-Induced NLRP3 Inflammasome Formation and Activation in Podocytes}

To further test whether AGEs play an important role in the formation and activation of NLRP3 inflammasome induced by D-ribose, AG, an AGEs formation inhibitor and alagebrium chloride (ALT), an AGEs breaker were used prior to incubation with D-ribose or vehicle. As shown in Figure 5, co-localization of NLRP3 with ASC (Figures 5A,C) or caspase-1 (Figures 5B,D) was much higher in D-ribose-treated podocytes than Vehlor L-ribose treated podocytes, but similar to D-glucose-treated podocytes. However, prior treatment of podocytes with AG or ALT completely blocked D-ribose or D-glucose-induced colocalization of NLRP3 with ASC or caspase-1. The level of cleaved caspase-1 (Figures 5E,F), its activity (Figure 5G) and IL-1 $\beta$ production (Figure $5 \mathbf{H}$ ) were remarkably elevated by D-ribose treatment, which was completely blocked by prior treatment of podocytes with AG or ALT. AG or ALT was also found to attenuate D-ribose-induced increased expression of AGEs and RAGE in podocytes (Figures 6A-D). D-glucose had a similar effect to D-ribose on NLRP3 inflammasome activation in podocytes, which was blocked by prior treatment of AG or ALT.

\section{RNA Interference of RAGE Prevented D-Ribose-Induced NLRP3 Inflammasome Formation and Activation in Podocytes}

We further examined the role of RAGE in D-ribose-induced NLRP3 inflammasome formation and activation. In these experiments, a siRNA against RAGE (siRAGE) was transfected to podocytes prior to administration of D-ribose or controls. This siRNA effectively silenced RAGE expression and blocked D-ribose-induced increase in RAGE expression as shown by Western blot analysis (Figures 7A-C). By confocal microscopy, co-localization of NLRP3 (green) with ASC (red) or caspase1 (red) was demonstrated to be much higher in D-ribosetreated podocytes than Vehl- or L-ribose-treated podocytes. RAGE gene silencing completely blocked D-ribose-induced colocalization of NLRP3 with ASC or caspase-1 (Figures 8A-D). RAGE gene silencing markedly diminished D-ribose-induced increase in cleaved caspase-1 levels (Figures 8E,G), caspase-1 activity (Figure 8F) and IL-1 $\beta$ production (Figure $8 \mathrm{H}$ ). Similarly, D-glucose-induced NLRP3 inflammasome formation and activation in podocytes were also attenuated by RAGE gene silencing.

\section{DISCUSSION}

The present study was designed to determine whether D-ribose induces NLRP3 inflammasome activation and results in podocyte and glomerular injuries and whether it depends upon AGEsRAGE signaling pathway. It was found that D-ribose induced NLRP3 inflammasome formation and activation, which led to podocyte injury and glomerular sclerosis in WT mice. Knockout of gene encoding Asc blocked D-ribose-induced NLRP3 inflammasome activation and consequent podocyte injury in mouse glomeruli. Furthermore, in vitro studies using cultured podocytes showed that D-ribose indeed stimulated NRLP3 inflammasome formation and activation. Inhibition of AGEs formation, increases in AGEs cleavage or silencing of RAGE gene all prevented D-ribose-induced NLRP3 inflammasome formation and activation, which protected podocytes from D-ribose-induced injury. These findings suggest that activation 
A

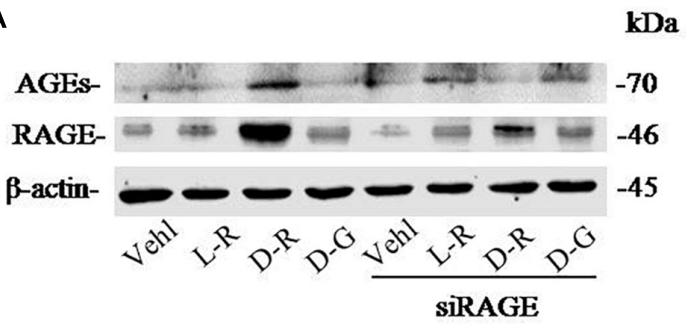

B

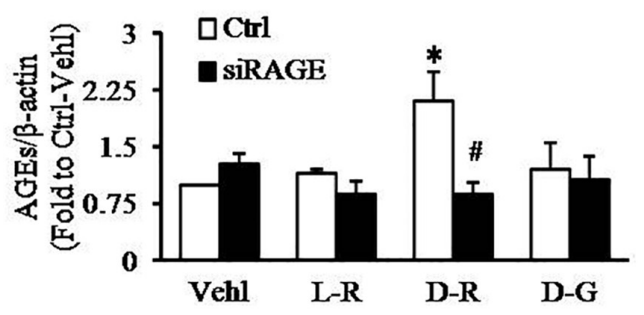

c

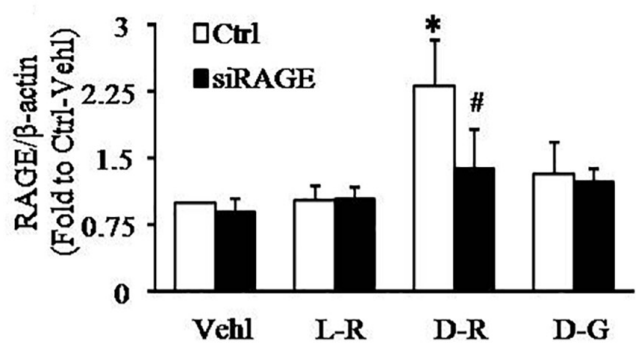

FIGURE 7 | RNA interference of RAGE prevented D-ribose-induced NLRP3 inflammasome formation and activation in podocytes. (A) Representative western blot gel document of AGEs and RAGE in podocytes with different treatments $(n=6)$. (B) Summarized data of AGEs in podocytes with different treatments $(n=6)$. (C) Summarized data of RAGE in podocytes with different treatments $(n=6)$. ${ }^{*} P<0.05$ versus Ctrl-Vehl group, ${ }^{\#} P<0.05$ versus Ctrl group. Ctrl, control; Vehl, Vehicle; L-R, L-ribose; D-R, D-ribose; D-G, D-glucose.

of RAGE by AGEs plays a crucial role in NLRP3 inflammasome activation and podocyte injury induced by $\mathrm{D}$-ribose.

Diabetes mellitus is characterized by high blood glucose levels due to either insulin deficiency or insulin resistance, which generates many organ damages leading to degenerative complications such as diabetic retinopathy, and renal glomerular injuries. Although increased blood D-glucose level is widely known to be responsible for the development of diabetic complications, D-ribose is now emerging as a novel pathogenic factor for organ damages during DM. However, the molecular mechanism mediating the pathogenic action of D-ribose remains poorly understood. In the present study, we first demonstrated that i.p. injection of D-ribose induced podocyte injury and glomerular sclerosis in mice, which were associated with NLRP3 inflammasome activation and consequent increase in IL-1 $\beta$ production, suggesting that NLRP3 inflammasome activation may be a critical mechanism for D-ribose-induced podocyte injury and progressive development of glomerular sclerosis. In Asc gene knockout mice, D-ribose failed to produce NLRP3 inflammasome activation, which prevented D-ribose-induced podocyte injury and glomerular sclerosis. This further confirms that NLRP3 inflammasome activation is crucial for the pathogenic action of D-ribose. To our knowledge, these findings provide the first experimental evidence that D-ribose may induce podocyte injury and glomerular sclerosis via activation of the NLRP3 inflammasome. Recently, it has been reported that NLRP3 inflammasome activation serves as a triggering mechanism leading to glomerular injury and ultimate end-stage renal disease (ESRD) under different pathological conditions (Yi et al., 2006; Abais et al., 2014a). In this regard, there is evidence that the expression of NLRP3 inflammasome molecules and proinflammatory cytokines increased in patients and mice suffering from T2DM (Vandanmagsar et al., 2011; Lee et al., 2013). NLRP3 or caspase-1 gene knockout and caspase-1 inhibition blocked or even reversed the progression diabetic nephropathy in mice (Wen et al., 2011). All these results suggest that the NLRP3 inflammasome may be an important pathogenic factor for the development of diabetic nephropathy, which is probably a new target for treatment of DN complications in the kidney (Segelmark and Hellmark, 2010). Given that NLRP3 inflammasome activation may be initiated by the production of mitochondrial reactive oxygen species (ROS) and increased NADPH oxidase activity during DM (Gao et al., 2015), the action of D-ribose to activate NLRP3 inflammasome may be associated with this redox regulation because it can react with $\beta_{2}$-microglobulin and induces the ribosylated protein through a ROS-mediated pathway (Kong et al., 2011).

As a reducing saccharide, D-ribose reacts with amino groups to initiate protein glycation and leads to the formation of AGEs, a type of major diabetic detrimental mixtures (Han et al., 2011). Previous studies showed that D-ribose induced AGEs accumulation (Han et al., 2011, 2014; Wei et al., 2012) and resulted in impairment of renal function (Wendt et al., 2003; Yeh et al., 2017). D-ribose is a stronger reducing sugar than D-glucose and it produces AGEs much more rapidly than D-glucose (Chen et al., 2009). In previous studies, the interaction between AGEs and RAGE has been confirmed to activate tissue inflammation and cell damages under pathological conditions, in particular, during DM (Chuah et al., 2013). However, it remains unknown whether AGEs-RAGE signaling pathway is involved in D-riboseinduced NLRP3 inflammasome activation and thereby triggers the inflammatory response in tissue or organs. The present study indeed confirmed the increased level of both AGEs and RAGE in glomeruli of mice treated with D-ribose, which could be blocked by prior treatment of AGEs formation inhibitor, AG. But whether AG could directly inhibit NLRP3 inflammasome formation needs further study. In cultured podocytes, we also found that D-ribose produced more AGEs compared to D-glucose. This led us to hypothesize that D-ribose may increase AGEs formation and thereby activate RAGE to produce NLRP3 inflammasome activation and consequent podocyte injury.

To test this hypothesis, we performed three series of cell studies to examine whether inhibition of AGEs formation, cleavage of AGE-derived protein crosslinks and gene silencing of AGEs receptor - RAGE attenuate or block D-ribose-induced NLRP3 inflammasome formation and activation. First, we demonstrated that D-ribose indeed induced NLRP3 inflammasome formation 
A

NIIRP3/ASC
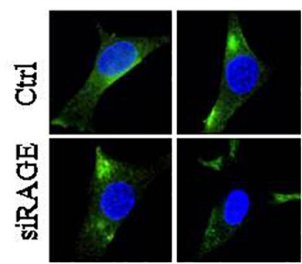

Vehl

C

NLIRP3/ASC

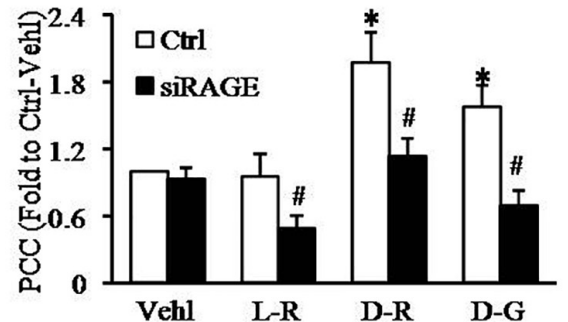

E

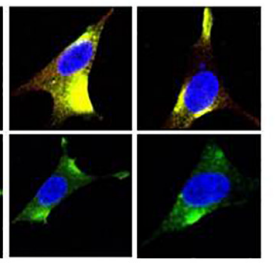

D-R D-G

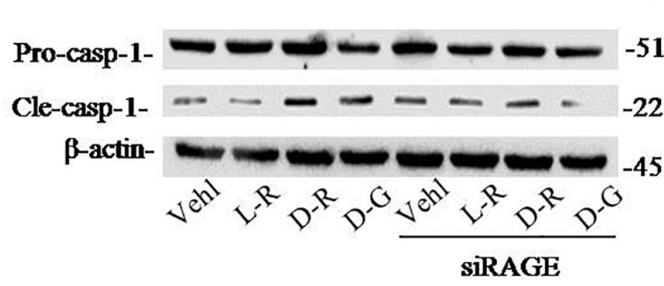

G

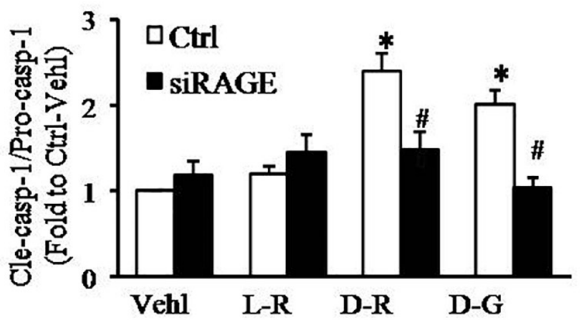

B NLJRP3/Caspase-1

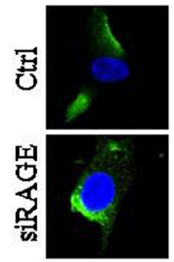

Vehl
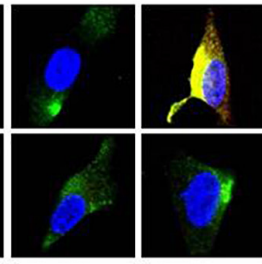

D-R

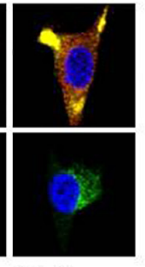

D-G

D NIJRP3/Caspase-1

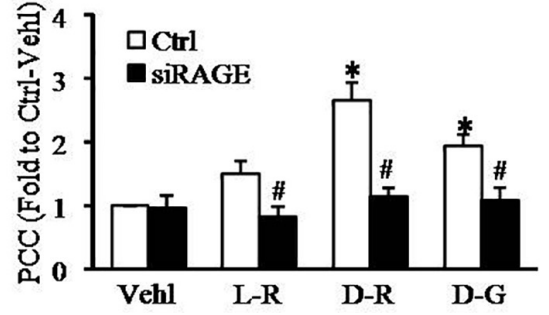

F

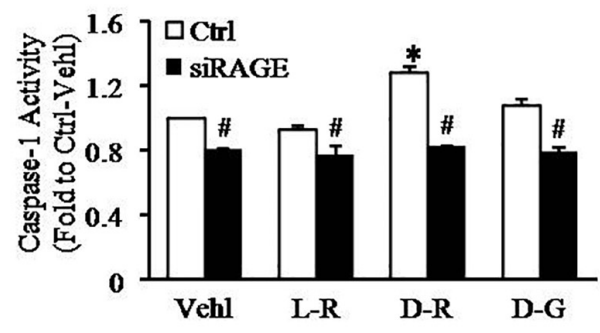

H

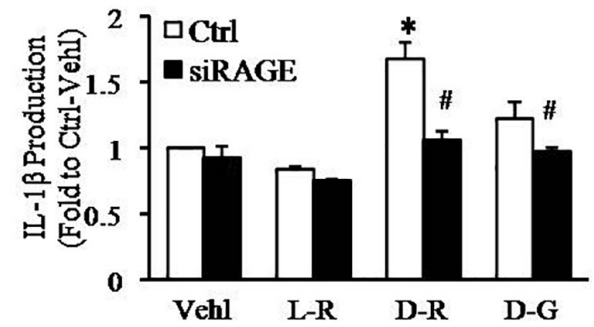

FIGURE 8 | RNA interference of RAGE prevented D-ribose-induced NLRP3 inflammasome formation and activation in podocytes. (A) Representative confocal microscopic images showing co-localization of NLRP3 (green) with ASC (red) $(n=6)$. (B) Representative confocal microscopic images showing co-localization of NLRP3 (green) with caspase-1 (red) $(n=6)$. (C) Summarized data depicting co-localization of NLRP3 (green) with ASC (red) during different treatments of podocytes $(n=6)$. (D) Summarized data depicting co-localization of NLRP3 (green) with caspase-1 (red) during different treatments of podocytes $(n=6)$. (E) Representative Western blot gel document of pro-Caspase-1 and Cle-Caspase-1 in podocytes with different treatments $(n=5)$. (F) Caspase-1 activity in podocytes with different treatments $(n=5)$. (G) Summarized data of pro-Caspase- 1 and Cle-Caspase- 1 in podocytes with different treatments $(n=5)$. (H) IL-1 $\beta$ levels in the supernatant of podocyte cultures with different treatments $(n=5) .{ }^{*} P<0.05$ versus Ctrl-Vehl group, ${ }^{\#} P<0.05$ versus Ctrl group. Ctrl, control; Vehl, Vehicle; L-R, L-ribose; $D-R$, D-ribose; D-G, D-glucose.

and activation in podocytes, because the inhibition of caspase-1 activity almost completely blocked D-ribose-induced NLRP3 inflammasome formation and activation. This effect of D-ribose was similar to glucose confirmed in previous studies in vitro (Abais et al., 2013, 2014a), indicating that a reducing sugar is a strong trigger of the NLRP3 inflammasome.

Second, we confirmed that either inhibition of AGEs formation by AG or cleavage of AGE-derived protein crosslinks by ALT substantially blocked D-ribose-induced NLRP3 inflammasome formation and activation, which was similar to the effect of caspase-1 inhibition. This suggests that increased AGEs may mediate the action of D-ribose to activate NLRP3 inflammasome in podocytes. Interestingly, L-ribose seems to have some response in cle-caspase-1 which was not inhibited by AG, but inhibited by ALT. How L-ribose works with the formation or cleavage of AGEs needs further studies. Although 
there is no direct evidence in literature showing the role of AGEs in mediating D-ribose-induced activation of the NLRP3 inflammasome in the kidney, some previous studies did confirm that D-ribose increased the level of AGEs in cultured human kidney 293 cells, human neuroblastoma SH-SY5Y cells, and primary cultured hippocampal neurons (Wei et al., 2009). Longterm administration of AGEs induced NLRP3 inflammasome activation and chronic renal injury (Yeh et al., 2017). In the context of AGEs activation of the NLRP3 inflammasome, there are reports that AGEs may serve as a pathogenic factor to activate NLRP3 or other inflammasome under different pathological conditions such as aging, DM, atherosclerosis (Kong et al., 2017; Deng et al., 2018). In some phagocytes such as polarized macrophages, however, AGEs significantly dampened NLRP3 inflammasome activation stimulated by influenza virus infection. It seems AGEs regulation of NLRP3 inflammasome activity depends upon tissue and cell types, which will need to be further investigated in future studies.

Finally, we tested whether RAGE is critically involved in NLRP3 inflammasome activation induced by D-ribose in podocytes. RAGE gene silencing completely blocked D-riboseinduced formation and activation of the NLRP3 inflammasome in podocytes. Based on these results, it appears that normal or increased RAGE expression is essential for D-riboseinduced NLRP3 inflammasome activation via increased AGEs. In previous studies, the role of AGEs-RAGE interaction in both diabetic nephropathy and nondiabetic renal diseases has been extensively studied and it is well accepted that AGERAGE signaling pathway is crucial for renal or glomerular inflammation, a hallmark of chronic kidney diseases (Abel et al., 1995; Tanji et al., 2000; Wendt et al., 2003; Che et al., 2011). For example, RAGE is expressed on normal podocytes and is upregulated in diabetic nephropathy (Tanji et al., 2000), which may drive the development of glomerular sclerosis (Wendt et al., 2003). Suppression of AGEs-RAGE pathway protected the kidney from pro-inflammatory injury, in particular, in renal glomeruli during DM (Wendt et al., 2003; Chow et al., 2006; Che et al., 2011) and antagonists of RAGE inhibited AGEs-induced NLRP3 inflammasome activation and consequent chronic renal injury (Yeh et al., 2017).

\section{CONCLUSION}

In summary, the present study demonstrated that chronic administration of D-ribose induced NLRP3 inflammasome

\section{REFERENCES}

Abais, J. M., Xia, M., Li, G., Chen, Y., Conley, S. M., Gehr, T. W., et al. (2014a). Nod-like receptor protein 3 (NLRP3) inflammasome activation and podocyte injury via thioredoxin-interacting protein (TXNIP) during hyperhomocysteinemia. J. Biol. Chem. 289, 27159-27168. doi: 10.1074/jbc.M114.56 7537

Abais, J. M., Xia, M., Li, G., Gehr, T. W., Boini, K. M., and Li, P. L. (2014b). Contribution of endogenously produced reactive oxygen species to the activation of podocyte NLRP3 inflammasomes in hyperhomocysteinemia. activation in podocytes and glomeruli, which was mediated by AGEs-RAGE signaling pathway. These findings elucidate a novel mechanism mediating D-ribose-induced podocyte injury and glomerular sclerosis, providing new insights into the pathogenesis of diabetic nephropathy, which may help identify D-ribose and its pathogenic action as a new therapeutic target for the treatment and prevention of diabetic nephropathy.

\section{DATA AVAILABILITY STATEMENT}

The data used to support the findings of this study are available from the corresponding author upon request.

\section{ETHICS STATEMENT}

The studies involving animal study was reviewed and approved by the Institutional Animal Care and Use Committee of the Virginia Commonwealth University.

\section{AUTHOR CONTRIBUTIONS}

P-LL, JR, and WL contributed to the conception of the study. JH, GL, and QZ contributed significantly to analysis and manuscript preparation. $\mathrm{JH}$ and GL performed the data analyses and wrote the manuscript. JH, GL, and P-LL helped perform the analysis with constructive discussions.

\section{FUNDING}

This study was supported by grants DK054927 and HL075316 from National Institutes of Health and grants 81573763 and 81530099 from National Natural Science Foundation of China.

\section{ACKNOWLEDGMENTS}

We acknowledge the support received by Jinni Hong from the China Scholarship Council (CSC) for visiting Ph.D. students (CSC No. 201706010324).

Free Radic. Biol. Med. 67, 211-220. doi: 10.1016/j.freeradbiomed.2013. 10.009

Abais, J. M., Zhang, C., Xia, M., Liu, Q., Gehr, T. W., Boini, K. M., et al. (2013). NADPH oxidase-mediated triggering of inflammasome activation in mouse podocytes and glomeruli during hyperhomocysteinemia. Antioxid. Redox. Signal. 18, 1537-1548. doi: 10.1089/ars.2012. 4666

Abel, M., Ritthaler, U., Zhang, Y., Deng, Y., Schmidt, A. M., Greten, J., et al. (1995). Expression of receptors for advanced glycosylated end-products in renal disease. Nephrol. Dial. Transplant. 10, 1662-1667. 
Babaei-Jadidi, R., Karachalias, N., Ahmed, N., Battah, S., and Thornalley, P. J. (2003). Prevention of incipient diabetic nephropathy by high-dose thiamine and benfotiamine. Diabetes Metab. Res. Rev. 52, 2110-2120. doi: 10.2337/ diabetes.52.8.2110

Bakker, P. J., Butter, L. M., Kors, L., Teske, G. J., Aten, J., Sutterwala, F. S., et al. (2014). Nlrp3 is a key modulator of diet-induced nephropathy and renal cholesterol accumulation. Kidney Int. 85, 1112-1122. doi: 10.1038/ki. 2013.503

Che, J., Okigaki, M., Takahashi, T., Katsume, A., Adachi, Y., Yamaguchi, S., et al. (2011). Endothelial FGF receptor signaling accelerates atherosclerosis. Am. J. Physiol. Heart Circ. Physiol. 300, H154-H161. doi: 10.1152/ajpheart.00075. 2010

Chen, L., Wei, Y., Wang, X., and He, R. (2009). D-Ribosylated Tau forms globular aggregates with high cytotoxicity. Cell. Mol. Life Sci. 66, 2559-2571. doi: 10. 1007/s00018-009-0058-57

Chow, F. Y., Nikolic-Paterson, D. J., Ozols, E., Atkins, R. C., Rollin, B. J., and Tesch, G. H. (2006). Monocyte chemoattractant protein-1 promotes the development of diabetic renal injury in streptozotocin-treated mice. Kidney Int. 69, 73-80. doi: 10.1038/sj.ki.5000014

Chowdhury, P. (2017). Aminoguanidine (AG) induces induced both pro- and antioxidant effect in AR42J cells, a rat pancreatic tumor cell line. Ann. Clin. Lab. Sci. 47, 572-580.

Chuah, Y. K., Basir, R., Talib, H., Tie, T. H., and Nordin, N. (2013). Receptor for advanced glycation end products and its involvement in inflammatory diseases. Int. J. Inflam. 2013:403460. doi: 10.1155/2013/403460

Day, J. F., Thorpe, S. R., and Baynes, J. W. (1979). Nonenzymatically glucosylated albumin. In vitro preparation and isolation from normal human serum. J. Biol. Chem. 254, 595-597.

Deng, X., Huang, W., Peng, J., Zhu, T. T., Sun, X. L., Zhou, X. Y., et al. (2018). Irisin alleviates advanced glycation end products-induced inflammation and endothelial dysfunction via inhibiting ROS-NLRP3 inflammasome signaling. Inflammation 41, 260-275. doi: 10.1007/s10753-017-068 5-683

Dhar, A., Dhar, I., Bhat, A., and Desai, K. M. (2016). Alagebrium attenuates methylglyoxal induced oxidative stress and AGE formation in H9C2 cardiac myocytes. Life Sci. 146, 8-14. doi: 10.1016/j.lfs.2016. 01.006

Gao, P., He, F. F., Tang, H., Lei, C. T., Chen, S., Meng, X. F., et al. (2015). NADPH oxidase-induced NALP3 inflammasome activation is driven by thioredoxin-interacting protein which contributes to podocyte injury in hyperglycemia. J. Diabetes Res. 2015:504761. doi: 10.1155/2015/50 4761

Gross, M., Reiter, S., and Zollner, N. (1989). Metabolism of D-ribose administered continuously to healthy persons and to patients with myoadenylate deaminase deficiency. Klin. Wochenschr. 67, 1205-1213. doi: 10.1007/bf0171 6208

Han, C., Lu, Y., Wei, Y., Liu, Y., and He, R. (2011). D-ribose induces cellular protein glycation and impairs mouse spatial cognition. PLoS One 6:e24623. doi: 10.1371/journal.pone.0024623

Han, C., Lu, Y., Wei, Y., Wu, B., Liu, Y., and He, R. (2014). D-ribosylation induces cognitive impairment through RAGE-dependent astrocytic inflammation. Cell Death Dis. 5:e1117. doi: 10.1038/cddis.2014.89

Hong, J., Bhat, O. M., Li, G., Dempsey, S. K., Zhang, Q., Ritter, J. K., et al. (2019). Lysosomal regulation of extracellular vesicle excretion during d-ribose-induced NLRP3 inflammasome activation in podocytes. Biochim. Biophys. Acta Mol. Cell. Res. 1866, 849-860. doi: 10.1016/j.bbamcr.2019. 02.007

Kong, F. L., Cheng, W., Chen, J., and Liang, Y. (2011). d-Ribose glycates beta(2)microglobulin to form aggregates with high cytotoxicity through a ROSmediated pathway. Chem. Biol. Interact. 194, 69-78. doi: 10.1016/j.cbi.2011. 08.003

Kong, X., Lu, A. L., Yao, X. M., Hua, Q., Li, X. Y., Qin, L., et al. (2017). Activation of NLRP3 inflammasome by advanced glycation end products promotes pancreatic islet damage. Oxid. Med. Cell. Longev. 2017:9692546. doi: $10.1155 / 2017 / 9692546$

Lee, H. M., Kim, J. J., Kim, H. J., Shong, M., Ku, B. J., and Jo, E. K. (2013). Upregulated NLRP3 inflammasome activation in patients with type 2 diabetes. Diabetes Metab. Res. Rev. 62, 194-204. doi: 10.2337/db12-0420
Lopez-Clavijo, A. F., Duque-Daza, C. A., Romero, C. I., Barrow, M. P., Kilgour, D., Rabbani, N., et al. (2014). Study of an unusual advanced glycation end-product (AGE) derived from glyoxal using mass spectrometry. J. Am. Soc. Mass Spectrom. 25, 673-683. doi: 10.1007/s13361-013-079 9-792

Martinon, F., and Tschopp, J. (2005). NLRs join TLRs as innate sensors of pathogens. Trends Immunol. 26, 447-454. doi: 10.1016/j.it.2005. 06.004

Perlmutter, N. S., Wilson, R. A., Angello, D. A., Palac, R. T., Lin, J., and Brown, B. G. (1991). Ribose facilitates thallium-201 redistribution in patients with coronary artery disease. J. Nucl. Med. 32, 193-200.

Raij, L., Azar, S., and Keane, W. (1984). Mesangial immune injury, hypertension, and progressive glomerular damage in Dahl rats. Kidney Int. 26, 137-143. doi: 10.1038/ki.1984.147

Segelmark, M., and Hellmark, T. (2010). Autoimmune kidney diseases. Autoimmun. Rev. 9, A366-A371. doi: 10.1016/j.autrev.2009.11.007

Seifert, J. G., Subudhi, A. W., Fu, M. X., Riska, K. L., John, J. C., Shecterle, L. M., et al. (2009). The role of ribose on oxidative stress during hypoxic exercise: a pilot study. J. Med. Food 12, 690-693. doi: 10.1089/jmf.2008. 0065

Su, T., and He, R. (2014). D-ribose, an overlooked player in type 2 diabetes mellitus? Sci. China Life Sci. 57:361. doi: 10.1007/s11427-014-4614-4615

Tanji, N., Markowitz, G. S., Fu, C., Kislinger, T., Taguchi, A., Pischetsrieder, M., et al. (2000). Expression of advanced glycation end products and their cellular receptor RAGE in diabetic nephropathy and nondiabetic renal disease. J. Am. Soc. Nephrol. 11, 1656-1666.

Teitelbaum, J. E., Johnson, C., and St Cyr, J. (2006). The use of D-ribose in chronic fatigue syndrome and fibromyalgia: a pilot study. J. Altern. Complement. Med. 12, 857-862. doi: 10.1089/acm.2006.12.857

Tessier, F. J., Niquet-Leridon, C., Jacolot, P., Jouquand, C., Genin, M., Schmidt, A. M., et al. (2016). Quantitative assessment of organ distribution of dietary protein-bound (13) C-labeled $\mathrm{N}$ (varepsilon) -carboxymethyllysine after a chronic oral exposure in mice. Mol. Nutr. Food Res. 60, 2446-2456. doi: 10. 1002/mnfr.201600140

Vandanmagsar, B., Youm, Y. H., Ravussin, A., Galgani, J. E., Stadler, K., Mynatt, R. L., et al. (2011). The NLRP3 inflammasome instigates obesity-induced inflammation and insulin resistance. Nat. Med. 17, 179-188. doi: 10.1038/nm. 2279

Wei, Y., Chen, L., Chen, J., Ge, L., and He, R. Q. (2009). Rapid glycation with D-ribose induces globular amyloid-like aggregations of BSA with high cytotoxicity to SH-SY5Y cells. BMC Cell Biol. 10:10. doi: 10.1186/1471-212110- 10

Wei, Y., Han, C. S., Zhou, J., Liu, Y., Chen, L., and He, R. Q. (2012). D-ribose in glycation and protein aggregation. Biochim. Biophys. Acta 1820, 488-494. doi: 10.1016/j.bbagen.2012.01.005

Wen, H., Gris, D., Lei, Y., Jha, S., Zhang, L., Huang, M. T., et al. (2011). Fatty acid-induced NLRP3-ASC inflammasome activation interferes with insulin signaling. Nat. Immunol. 12, 408-415. doi: 10.1038/ni. 2022

Wendt, T. M., Tanji, N., Guo, J., Kislinger, T. R., Qu, W., Lu, Y., et al. (2003). RAGE drives the development of glomerulosclerosis and implicates podocyte activation in the pathogenesis of diabetic nephropathy. Am. J. Pathol. 162, 1123-1137. doi: 10.1016/S0002-9440(10)63909-63900

Wu, B., Wei, Y., Wang, Y., Su, T., Zhou, L., Liu, Y., et al. (2015). Gavage of D-Ribose induces Abeta-like deposits, Tau hyperphosphorylation as well as memory loss and anxiety-like behavior in mice. Oncotarget 6, 34128-34142. doi: 10.18632/oncotarget.6021

Yang, F., Wang, Z., Wei, X., Han, H., Meng, X., Zhang, Y., et al. (2014). NLRP3 deficiency ameliorates neurovascular damage in experimental ischemic stroke. J. Cereb. Blood Flow Metab. 34, 660-667. doi: 10.1038/jcbfm.2013.242

Yavuz, D. G., Ersoz, H. O., Tuncel, M., Sargon, M. F., Kucukkaya, B., Ahiskali, R., et al. (2001). Effects of aminoguanidine on glomerular basement membrane thickness and anionic charge in a diabetic rat model. Int. J. Exp. Diabetes Res. 2, 225-232. doi: 10.1155/edr.2001.225

Yeh, W. J., Yang, H. Y., Pai, M. H., Wu, C. H., and Chen, J. R. (2017). Long-term administration of advanced glycation end-product stimulates the activation of NLRP3 inflammasome and sparking the development of renal injury. J. Nutr. Biochem. 39, 68-76. doi: 10.1016/j.jnutbio.2016.09.014 
Yi, F., Zhang, A. Y., Li, N., Muh, R. W., Fillet, M., Renert, A. F., et al. (2006). Inhibition of ceramide-redox signaling pathway blocks glomerular injury in hyperhomocysteinemic rats. Kidney Int. 70, 88-96. doi: 10.1038/sj.ki.5001517

Yin, Y., Pastrana, J. L., Li, X., Huang, X., Mallilankaraman, K., Choi, E. T., et al. (2013). Inflammasomes: sensors of metabolic stresses for vascular inflammation. Front. Biosci. 18, 638-649.

Zhang, C., Boini, K. M., Xia, M., Abais, J. M., Li, X., Liu, Q., et al. (2012). Activation of Nod-like receptor protein 3 inflammasomes turns on podocyte injury and glomerular sclerosis in hyperhomocysteinemia. Hypertension 60, 154-162. doi: 10.1161/HYPERTENSIONAHA.111.189688
Conflict of Interest: The authors declare that the research was conducted in the absence of any commercial or financial relationships that could be construed as a potential conflict of interest.

Copyright $\odot 2019$ Hong, Li, Zhang, Ritter, Li and Li. This is an open-access article distributed under the terms of the Creative Commons Attribution License (CC BY). The use, distribution or reproduction in other forums is permitted, provided the original author(s) and the copyright owner(s) are credited and that the original publication in this journal is cited, in accordance with accepted academic practice. No use, distribution or reproduction is permitted which does not comply with these terms. 